\title{
MONARQUÍA Y PARLAMENTO EN LA IUSPUBLICÍSTICA ITALIANA DE LA PRIMERA POSGUERRA*
}

FULCO LANCHESTER** 


\section{SUMARIO}

1. INTRODUCCIÓN.- 2. LA DERIVACIÓN DEL EstatUTO.- 3. DOCTRINA IUSPUBLICÍSTICA DE La PRIMERa posguerra.- 4. Monarquía y parlamento en la fase de deMOCRATIzación: La posición de Donato Donati Y GIOVANNI Salemi.- 5. (SIGUe): Gaspare Ambrosini y Emilio Crosa.- 6. Transición al régimen aUtoRITARIO ENTRE REGRESO AL PASADO Y NUEVOS PARADIGMAS: LA CONTRIBUCIÓN DE TEOdOSIO MARCHI.- 7. Sin VUELTA ATRÁS: LAS TRANSFORMACIONES DEL RÉGIMEN Y EL PAPEL DE LA MONARQUÍA.- 8. CONCLUSIONES. 


\title{
MONARQUÍA Y PARLAMENTO EN LA IUSPUBLICÍSTICA ITALIANA DE LA PRIMERA POSGUERRA*
}

POR

\author{
FULCO LANCHESTER**
}

\section{INTRODUCCIÓN}

Monarquía y Parlamento constituyen los polos clásicos de una relación institucional que ha dado vida a la misma discusión moderna sobre formas de Estado y formas de gobierno, mientras los iuspublicistas constituyen en cambio un grupo de especialistas que, en la división del trabajo ideológico del Estado nacional centralizado de origen decimonónico, han contribuido a crear, reproducir e interpretar paradigmas capaces de asumir un sentido pragmático, pero estrechamente ligados con situaciones precisas de la clase dirigente. En esta ponencia intentaré evidenciar la forma geométrica, pero variable, del triángulo que une las antedichas instituciones (monarquía y parlamento) a la iuspublicística italiana en un periodo crucial como el que discurre entre 1918 y 1926, en el que la crisis del Estado liberal oligárquico bajo los golpes de la participación popular da vida, en el lapso de pocos años, a la solución liberal democrática, por una parte, y a la autoritaria, por otro.

* Ponencia presentada en la Sexta Jornada de estudio "Luigi Luzzatti» para la historia de la Italia contemporánea Los juristas y la crisis del Estado liberal (1918-1926), 17-18 de noviembre de 2000.

** Traducción de Pedro J. Tenorio Sánchez, Profesor Titular de Derecho Constitucional de la UNED. 
Dejo en el trasfondo la dilucidación conceptual de los diversos significados de los dos términos institucionales a que me refiero, que cambian en el tiempo y deberían ser especificados cuidadosamente para no incurrir en peligrosas incomprensiones en relación con diferentes teorías de las relaciones institucionales. Intentaré en cambio concretar de manera sintética la realidad cuantitativa y cualitativa de la doctrina iuspublicística en el periodo cubierto por este congreso.

Por lo que se refiere al primer punto, basta -en efecto- pensar en la necesaria distinción entre la persona del Rey y la Corona como institución en el seno de la institución monárquica; en las diferentes especificaciones del término monarquía que derivan de los adjetivos "constitucional", "pura", "representativa", "parlamentaria", capaces de describir diferentes formas de Estado y de gobierno; en el contenido variable que los mismos conceptos de "Ejecutivo" y "Legislativo" tienen en el tiempo; $y$, por último, en la influencia sobre las clases dirigentes del siglo XIX de la teoria británica del «Rey en Parlamento".

Por lo que se refiere al segundo, considero indispensable proporcionar elementos sobre la posición de la doctrina iuspublicística acompañándola con datos concretos sobre sus dimensiones y articulaciones en el momento de la crisis del Estado liberal, a fin de evitar posibles malentendidos sobre la presencia de la llamada escuela iuspublicista nacional de derivación orlandiana ${ }^{1}$.

\section{LA DERIVACIÓN DEL ESTATUTO ALBERTINO}

El examen sistemático del tema monarquía-parlamento podría iluminar toda la evolución de la Italia estatutaria desde el periodo de la concesión de la Carta a la sucesiva parlamentarización del sistema sobre la base de convenciones².

1 Al respecto, v. M. GALIZIA, «Profili storico-comparativi della scienza del diritto costituzionale", en Archivio Filippo Serafini, 1963, pp. 1 ss.; G. CIANFEROT$\mathrm{TI}$, /I pensiero di V.E. Orlando e la giuspubblicistica italiana tra Ottocento e Novecento, Milano, Giuffrè, 1980; M. FioravantI, "Le dottrine dello Stato e della Costituzione", en R. Romanelu (director), Storia dello Stato italiano dall'Unità ad oggi, Roma, Donzelli, 1995, pp. 407 ss, y, últimamente, P. Grossı, Scienza giuridica italiana. Un profilo storico 1860-1950, Milano, Giuffrè, 2000.

2 El Estatuto fue otorgado por Carlos Alberto, Rey de Cerdeña, el 4 de marzo de 1848 y estuvo en vigor durante un siglo aproximadamente. 
De la escueta combinación de varios artículos del Estatuto $(3,5$, $65,67)$ con el preámbulo se dibujó, en los orígenes, una forma de gobierno monárquico-constitucional pura. Con gran celeridad la situación se modificó en un ordenamiento caracterizado por la conexión interorgánica y natural entre gobierno y parlamento. Todo esto tuvo lugar sobre la base de convenciones constitucionales, explotando la llamada flexibilidad del Estatuto. Durante los primeros cinco decenios de vigencia del mismo se evidenció, sin embargo, una tensión entre varias interpretaciones de la Carta y en particular entre la monárquicoconstitucional y la monárquico-parlamentaria, bajo la cobertura de la dicción estatutaria de la monarquía representativa ${ }^{3}$.

Es oportuno decir que, mientras los constitucionalistas del primer periodo de vigencia del Estatuto (en particular después de la proclamación del Reino), por la propia naturaleza de su labor, no pudieron sino preparar el camino de la aplicación de la Carta en sentido evolutivo y consuetudinario, sobre la base del ejemplo británico, los autores del periodo sucesivo o resolvieron los problemas a través de la ficción de la personalidad estatal, o abrazaron varios tipos de planteamientos que derivaban de criterios ligados a la función del instituto monárquico en el interior del Estado moderno y contemporáneo.

Ahora bien, más allá de toda reconstrucción doctrinal, resulta oportuno reconocer que el poder de la Corona hasta el final del primer conflicto mundial se desarrolla en el tiempo en correlación inversa con el del Parlamento y de las fuerzas políticas que están en el seno del mismo, dando vida a gabinetes más o menos estables. Después del repentino paso de la originaria forma constitucional pura a la parlamentaria el llamado poder de prerrogativa del monarca siguió presente no sólo en el ámbito del tradicional sector federativo (política exterior y política de la defensa), sino que se configuró como auténtico y propio poder de reserva en el seno del ordenamiento. Por esto algunos autores han localizado en el papel de la Corona y en particular en el del Monarca el elemento de reserva, que explica el desenvolvimiento no constitucional de la forma de gobierno parlamentaria ita-

3 El artículo 2 del Estatuto decía que «El Estado se rige por un Gobierno Monárquico representativo. El trono es hereditario de conformidad con la ley sálica". Para los varios tipos de reconstrucción, v. E CROSA, La monarchia nel diritto pubblico italiano, Torino, Bocca, 1922; y recientemente P. Coloмво, II re d'Italia: prerogative costituzionali e potere politico della Corona (1848-1922), Milano, Angeli, 1999 y el número especial de CHEIRON, La Monarchia nella storia d'Italia unita. Problematiche ed esemplificazioni, Roma, Bulzoni, 1996. 
liana. Desde Moncalieri hasta el 1898, para llegar al "mayo radiante» y al 1922 la crítica al comportamiento de la Corona ha sido decidida ${ }^{4}$, pero acaso en el análisis no se han tenido suficientemente en cuenta ni el dato técnico-constitucional ni las derivaciones que se presentaron en las opciones 5 .

\section{LA DOCTRINA IUSPUBLICISTA EN LA PRIMERA POSGUERRA}

Después de la primera guerra mundial la misma doctrina constitucionalista italiana se encontró ante el problema de metabolizar las novedades institucionales que habían irrumpido en el ordenamiento y de elaborar nuevos esquemas adecuados. Existía en muchos la sensación de que la envergadura de las innovaciones no podía ser considerada desde la continuidad, sino que éstas comportaban una auténtica y propia solución de continuidad, que se reflejaba en interpretaciones de conjunto del sistema.

La aplicación del sufragio universal masculino y la introducción de la llamada "proporcional» (el sistema electoral proporcional) no eran sino la punta de un iceberg de las novedades introducidas en el sistema. Francesco Ruffini ${ }^{6}$ en su intervención sobre Guerra y reformas

4 Nos referimos a cuatro momentos tópicos de la historia constitucional italiana del periodo estatutario. En el castillo de Moncalieri, el 20 de noviembre de 1849, el Rey Victorio Enmanuel II, por medio de una proclama, disuelve la Cámara de los Diputados, que se resistía a aprobar el Tratado de paz con Austria. En 1898 el Rey Umberto I fue el centro de intentos de resistencia a la democratización del sistema político-constitucional. En mayo de 1915 (llamado por la historiografía nacionalista "mayo radiante»), el Rey Victorio Enmanuel III asumió un papel decisivo favoreciendo la participación de Italia en el primer conflicto mundial. En octubre de 1922, la falta de proclamación del estado de sitio por parte de Victorio Enmanuel III se consideró como una ayuda objetiva al advenimiento de Mussolini al poder.

5 V.E. Rotelli, Costituzione e amministrazione dell'Italia unita, Bologna, II mulino, 1981; G. MARANINI, Storia del potere in Italia, Firenze, Vallecchi, 1967; G. PERTICONE, I/ regime parlamentare nella storia dello Statuto albertino, Roma, Edizioni dell'Ateneo, 1960; C. GHISALBERTI, Storia costituzionale d'Italia, Bari, Laterza, 1974; S. MerLININ, Autorità e democrazia nella forma di governo italiana, Torino, Giappichelli, 1997.

$6 \quad$ Francesco Ruffini, Senador del Reino, fue catedrático de Historia del Derecho en la Universidad de Turín y luego de 1910 a 1927 de Derecho eclesiástico. Con su hijo Eduardo Ruffini Avondo, Catedrático de Historia del Derecho italiano en la Universidad de Perugia, fue uno de los pocos catedráticos italianos que se negaron a jurar fidelidad al Fascismo. 
institucionales ${ }^{7}$ había abierto el camino a una reflexión a la que sin embargo sólo algunos entre los más atentos constitucionalistas habían prestado suficiente atención. Precisamente Gaspare Ambrosini, que había sido discípulo de Ruffini, en el pequeño volumen de 1921 sobre Partiti politici e gruppi parlamentari dopo la proporzionale ${ }^{8}$ comenzó a examinar seriamente las consecuencias de la introducción del sistema electoral basado en fórmula no mayoritaria y de las nuevas formaciones partidistas para la vida de la Asamblea de Montecitorio, mientras tanto Umberto Boss ${ }^{9}$ como Giovanni Salemi ${ }^{10}$ profundizaron en la Rivista di diritto pubblico las transformaciones del sector publicista11. Es de subrayar - por lo demás-que, precisamente en los inicios de 1922 , G. Capograssi había publicado el pequeño volumen sobre La nuova democrazia diretta ${ }^{12}$, significativa contribución de un cambio de paradigmas, en el que participaban tanto los filósofos del derecho como los historiadores del derecho ${ }^{13}$.

En aquellos mismos años los Maestros de la escuela iuspublicista nacional prefirieron, en cambio, continuar desarrollando los paradigmas tradicionales siguiendo volviendo la mirada a la doctrina alemana del periodo imperial, como demuestra la publicación precisamente en 1922 de la Dottrina dello Stato de Jellinek con larga intro-

7 V. F. RuFFINI, "Guerra e riforme istituzionali (Suffragio universale. Principio maggioritario. Elezione proporzionale. Rappresentanza organica)", en Annuario della R. Università di Torino, anno 1919-1920, pp. 5 s. Sobre esto v. Ampliamente N. ANTONETTI, Gli invalidi della Costituzione: il Senato del Regno 18481924, Roma-Bari, Laterza, 1992.

8 V. G. Ambrosinı, Partiti politici e gruppi parlamentari dopo la proporzionale, con un'appendice contenente il testo delle modifiche apportate il 26 luglio-6 agosto 1920 al Regolamento interno della Camera dei deputati, Firenze, La Voce, 1921.

9 Umberto Bossi fue catedrático de Derecho administrativo en la Universidad de Macerata entre 1906 y 1914, y luego en Pisa, Padua y Boloña.

10 Giovanni Salemi, profesor de Derecho administrativo desde 1922 en Sassari y Cagliari, fue profesor ordinario de la misma materia en Padua y Palermo.

$"$ V. U. Bossı, "Nuovi orientamenti e nuovi profili del diritto amministrativo italiano", en Rivista di diritto pubblico, 1920, I, pp. 1 ss.; G. SALEMI, "Il nuovo diritto pubblico e le sue caratteristiche fondamentali», en Rivista di diritto pubblico, 1921 , nn. 1-2, pp. 37 ss.

12 V. G. CAPOGRASSI, La nuova democrazia diretta, ahora en Opere, Milano, Giuffrè, 1990, vol. I, pp. 401 ss.

13 Significativa al respecto es la experiencia de G. Perticone, que transfirió al seno del ambiente publicístico su interés por la doctrina alemana: v. F. LANCHESTER, I giuspubblicisti tra storia e politica, Torino, Giappichelli, 1998, pp. 41 ss., pero también la de romanistas como Betti o de historiadores del Derecho como De Francisci. 
ducción de Vittorio Emanuele Orlando, obra que no por casualidad será reeditada precisamente en 1947, en correspondencia con el trabajo constituyente ${ }^{14}$.

De 1923 hasta el umbral de los años treinta la discusión nacional fue -en cambio- monopolizada primero por el proyecto Acerbo de introducción del premio de mayoría, luego por el tema de las reformas constitucionales (la llamada Commissione dei Soloni15) al que siguieron los primeros pasos hacia la construcción del régimen. En el ámbito iuspublicístico tales acontecimientos tendrán una influencia de tipo dicotómico. Desde el punto de vista institucional la formación de las Facultades de Ciencias Políticas - fruto de un debate intenso del periodo liberal modificado parcialmente por el Fascismo (pienso en las sedes de Roma y Pavia y en la militante de Perugia ${ }^{16}$ - - constituyó un acicate para la reflexión sobre las novedades derivadas de la crisis del Estado liberal-oligárquico, mientras en las Facultades de Derecho se enrocaron los exponentes de la vieja clase dirigente liberal.

Desde el punto de vista de la disciplina tuvo lugar - al menos en los años veinte- un singular reforzamiento de la escuela orlandiana en la selección académica, pero también la profundización de una diferenciación entre materias administrativas, en que los standard eran mucho más tradicionales, y las constitucionales, en las que será más evidente el debate sobre las novedades institucionales del régimen. Desde el punto de vista cuantitativo es también posible notar una reducción de los docentes catedráticos y no sólo como consecuencia del bloqueo de los concursos, sino sobre todo de la situación políticoconstitucional. En 1911 en Italia se contaban, en efecto 15 profesores numerarios de Derecho Constitucional ${ }^{17}$ y 20 de Derecho administrativo ${ }^{18}$; en $1922,13^{19}$ y $14^{20}$ respectivamente; en $1926,10^{21}$ y $17^{22}$.

14 V. G. JellineK, La dottrina dello Stato, trad. it. De la segunda edición alemana de M. Petrozziello con intr. $Y$ capítulos añadidos de V.E. Orlando, Milano, Società editrice libraria, 1921 ( $2^{\mathrm{a}}$ ed., Milano, Giuffrè, 1947).

15 Se trata de una Comisión nombrada por el Gobierno Mussolini y encargada de estudiar las reformas institucionales a introducir en Italia.

16 Las facultades de ciencias políticas estatales fueron fundadas a partir de 1925: la Facultad de Roma en 1925, la de Pavia en 1926, la de Perugia en 1927 (su denominación era Regia Facultad fascista de Ciencias políticas de Perugia).

17 Rossi, Chimienti, Donati, Arangio-Ruiz, Arcoleo, Morelli, Lombardo Pellegrino, Minguzzi, Orlando, Siotto Pintor, Romano, Luzzatti, Ferraccio, Marchi Mosca.

18 Mantovani Orsetti, Vitta, Raggi, Maiorana, Pacinotti, De Murtas Zichian, Bossi, Presutti, Persico, Ferraris, Longo, Tomassini, Ranelletti, Scalvanti, Bacchelli, Calandra, Soro Delitala, Leporini, Brondi, Nicolai Fiocchi. 
En el periodo sucesivo a 1922 iuspublicistas "realistas" como Emilio Crosa ${ }^{23}$ y Carmelo Caristia ${ }^{24}$ vieron reconocida su ideoneidad científica, mientras personajes militantes como Carlo Costamagna fueron rechazados por el establishment académico. En el periodo de los años treinta la cooptación académica englobó, en cambio, a personajes como Esposito ${ }^{25}$, Mortati26, Crisafulli27, o Chiarelli28, que no quisieron abandonar el cauce de la escuela iuspublicística nacional, aun cuando tomaran nota de las transformaciones de la época producidas precisamente con el proceso de masificación de las instituciones liberales oligárquicas.

19 Rossi, Chimienti, Lombardo Pellegrino, Arangio-Ruiz, Presutti, Donati, Siotto Pintor, Criscuoli, Romano, Luzzatti, Ferraccio, Mosca.

20 Bossi, Brondi, Cammeo, D'Alessio, Ferraris, Longo, Majorana, Pacinotti, Raggi, Ranelletti, Calandra, Solazzi, Tommasone, Vacchelli.

21 Siotto Pintor, Romano, Presutti, Donati, Ambrosini, Marchi, Orlando, Crosa, Criscuoli, Arangio Ruiz.

22 Bossi, Brondi, Cammeo, D'Alessio, Forti, Longo, Majorana, Raggi, Ranelleti, Calandra, Salemi, Solazzi, Tommasone, Vacchelli, Vitta, Zanobini.

23 Emilio Crosa, catedrático de Derecho constitucional en las Universidades de Pavia y de Turín.

24 Carmelo Caristia, profesor encargado en la Universidad de Macerata y luego catedrático de Instituciones de Derecho público y privado en el Instituto superior de ciencias económicas de Catania, que hasta los años treinta no dependía del Ministerio de instrucción pública, se hizo catedrático en la Facultad de Derecho de la misma Universidad en 1943 y luego diputado en la Asamblea constituyente en 1946.

25 Carlo Esposito, catedrático de Derecho constitucional y de Teoría del Estado en las Universidades de Macerata, Nápoles y Roma.

26 Constantino Mortati, profesor de Derecho constitucional en las Universidades de Messina y Macerata, enseñó en Nápoles y acabó su carrera académica como catedrático de Derecho constitucional italiano y comparado en la Facultad de Ciencias políticas de la Universidad de Roma. Fue elegido diputado de la Asamblea constituyente y luego nombrado juez del Tribunal Constitucional.

27 Vezio Crisafulli, catedrático de Derecho constitucional en las Universidades de Urbino, Trieste y Roma.

28 Giuseppe Chiarelli, catedrático de Derecho corporativo en Perugia, terminó su carrera académica como catedrático de Instituciones de Derecho público en la Facultad de Economía y comercio de la Universidad de Roma. Juez constitucional, llegó a ser Presidente del Tribunal Constitucional entre 1971 y 1973. 


\section{MONARQUÍA Y PARLAMENTO EN LA FASE DE DEMOCRATIZACIÓN: LA POSICIÓN DE DONATO DONATI Y GIOVANNI SALEMI.}

En el periodo ya final de la breve experiencia liberal-democrática, en el n. 1-2 de la Rivista di diritto pubblico e della pubblica amministrazione in Italia e giurisprudenza amministrativa esposta sistematicamente de 1921, dirigida por Luzzatti-Orlando-Salandra-Codacci Pisanelli-Calisse-Corradino y D'Amelio, fueron publicados los significativos escritos de dos constitucionalistas. En el primero Donato Donati29, catedrático en Padua, afrontaba la cuestión de La persona reale dello Stato; en el segundo, Giovanni Salemi, "profesor extraordinario" en Sassari, se dedicaba a II nuovo diritto pubblico e le sue caratteristiche fondamentali. En el momento en que buena parte de la iuspublicística se abstraía sustancialmente del debate o volvía a proponer las categorías de la doctrina jurídico-pública alemana sobre la base de las enseñanzas de Georg Jellinek ${ }^{30}$, las dos contribuciones eran extraordinariamente convergentes en los resultados, aun cuando profundamente diversas en el método. Donato Donati sostenía que «la sustancia de la persona real del Estado está formada por el conjunto de sus funcionarios en cuanto tales, es decir, de lo que suele llamarse la organización estatal ${ }^{31}$ ». Este tipo de planteamiento llevaba a Donati a especificar que la naturaleza de la persona estatal podía tener carácter de Institución o de corporación, según que los destinatarios o beneficiarios de su actividad fueran admitidos a participar en la organización de la persona colectiva ${ }^{32}$. "La naturaleza de simple institución o de corporación del Estado - para Donati- [acababa dependiendo] de la circunstancia de hecho de que el pueblo [fuera] o no admitido al ejercicio de la soberanía "y de la medida en que lo fuera»"33.

Tal construcción, en la que eran evidentes las sugerencias gierkianas servía a Donati para señalar que uen la forma de gobierno democrá-

29 Donato Donati, profesor de Derecho constitucional en Camerino y en Macerata, llegó a ser catedrático de la misma materia en la Facultad de Derecho de la Universidad de Padua.

30 V. G. JELLINEK, La dottrina generale dello Stato, cit. sobre la cual v. La recensión de N. COCO, "Giorgio Jellinek e la odierna crisi dello stato", en Rivista di diritto pubblico, 1923, I, pp. 42 ss.

31 V. D. DONATI, "La persona reale dello Stato", en Rivista di diritto pubblico, 1921, l, p. 10 y también pp. 15-16.

32 Idem, p. 16.

33 Ibídem, p. 17. 
tico [sic] (directo o representativo) propia del Estado moderno, el pueblo, además de ser objeto de la soberania, asume una posición ulterior, en cuanto los ciudadanos son admitidos al ejercicio de la soberanía" ${ }^{34}$, pero también para sostener que "los funcionarios públicos deben siempre ser considerados sustancia de la persona estatal». En este marco, que se mueve en el ámbito de los conceptos de la doctrina alemana del siglo anterior, acabando por superarla, el monarca es marginado porque «el pueblo alcanza en el ordenamiento del Estado aquella sustancial autonomía y potestad de autogobierno, por lo que él mismo verdaderamente puede considerarse árbitro del propio destino" ${ }^{35}$.

Giovanni Salemi, en su escrito, de corte más pragmático, afrontaba - en cambio - el conjunto de las innovaciones institucionales que "durante y después de la conflagración mundial» ${ }^{36}$ [habian sido] capaces de transformar la monarquía parlamentaria italiana uen una completa democracia»37. Concordando con los análisis de Gaspar Ambrosini sobre la superación del individualismo del periodo liberal, Salemi sostenía que, en el momento en que estaba escribiendo, colectivismo e individualismo "se ensamblan y se adecuan", entre otros aspectos sobre la base de la entrada de los partidos políticos, que «son reconocidos y a los que se conceden funciones públicas, y que contribuyen a manifestar la voluntad del Estado» ${ }^{38}$. La extensión del sufragio, la adopción del sistema proporcional y el nuevo papel de los grupos parlamentarios no constituían las únicas novedades, capaces de modificar e incidir en la concepción tradicional de la representación política regulada por el artículo 41 del Estatuto, sino que eran acompañadas por otras incisivas innovaciones precisamente en perjuicio de la Corona. Con el nuevo reglamento de 1920 (v. art. 3 del Reglamento del Senado) el Senado habia conseguido participar en el nombramiento del presidente y de los vicepresidentes de dicha Cámara con una modificación material del art. 35 del Estatuto, mientras la Cámara de los diputados había conquistado el derecho de auto-convocatoria, superando el art. 9 del Estatuto.

Salemi recordaba también las discusiones sobre la reforma del Senado y en particular los Dictámenes de la Comisión especial para la

34 Ibidem.

35 Ibídem, pp. 21-22.

36 V.G. SALEMI, "Il nuovo diritto pubblico e le sue caratteristiche fondamentali", en Rivista di diritto pubblico, cit., p. 37.

37 Idem, p. 39.

$38 \quad$ Ibídem, p. 42. 
reforma del Senado (Roma, 1919) de la que había sido magna pars Francesco Ruffini y los proyectos de la Cámara con relación a la declaración de guerra, a los tratados internacionales $y$ al ejercicio de la amnistía. Al final de un excursus que comprendía los ámbitos constitucional, administrativo y judicial, Salemi repetía que sobre la base de las transformaciones derivadas del conflicto "la voluntad del soberano se sustituye en nuestro País ... por la del Senado y la Cámara de los diputados. A la voluntad de los órganos ejecutivos o judiciales, antes nombrados por el Rey, se une el de individuos, elegidos por específicas categorías $u$ organizaciones de ciudadanos, siendo así reducida a una simple formalidad la intervención regia. La voluntad de todos los partidos políticos prevalece, mediante la representación proporcional sobre la de una minoría popular, que conseguía imponerse en las elecciones con sistema mayoritario» ${ }^{39}$.

La conclusión de Salemi era por tanto que «nuestro Derecho público [ha] aceptado con prontitud los ideales aparecidos durante $y$ después de la guerra; [ha] seguido la corriente de los mismos, en vez de ponerse en contran ${ }^{40}$.

\section{5. (sigue): GASPARE AMBROSINI Y EMILIO CROSA}

Una postura similar respecto del Parlamento, entendido como órgano de representación organizada de los intereses existentes en la sociedad política se podría encontrar de manera expresiva en Gaspare Ambrosini41, crítico y testigo de la crisis del Estado liberal oligárquico, que dedicó su atención precisamente al intento de una elaboración nueva dél tema de la representación política, así como a las nuevas tendencias hacia la representación de intereses.

En el Ensayo sobre Partiti e gruppi parlamentari dopo la proporzionale $^{42}$ Ambrosini demostraba haber comprendido que algo enorme

$39 \quad$ lbídem, p. 52.

$40 \quad$ lbidem, p. 64.

41 Gaspare Ambrosini, profesor de Derecho eclesiástico en la Universidad de Mesina (1912), se hizo en 1919 catedrático de Derecho constitucional de la Universidad de Palermo, y luego, en 1938, de Derecho colonial en la Universidad de Roma; acabó su carrera como catedrático de Derecho constitucional en la misma Universidad. Diputado en la Asamblea constituyente (1946), en los años cincuenta llegó a ser miembro del Tribunal Constitucional y luego su Presidente entre 1962 y 1967. nale, cit.

42 V. G. AmBrosini, Partiti politici e gruppi parlamentari dopo la proporzio- 
había sucedido en las estructuras del Estado liberal, con la necesidad de llevar a cabo las adaptaciones oportunas. El Rey y la Corona estaban en el trasfondo, mientras se repetían con fuerza y decisión la importancia de las reformas de la ley de elecciones generales y de los reglamentos parlamentarios "para la vida del régimen representativo parlamentario" en la medida en que incidian sobre la "base misma de la representación política", porque señalaban la entrada de los partidos políticos y de los grupos parlamentarios en la vida constitucional e intentaban corregir los defectos de la misma forma de gobierno ${ }^{43}$.

La parte más importante, en concreto para identificar los límites necesarios de la conceptualización de Ambrosini, está sin embargo localizable en el capítulo VIII del pequeño volumen en cuestión ${ }^{44}$. En éste Ambrosini examinaba tanto los críticas de la escuela iuspublicista nacional a la representación proporcional como proemio de un regreso peligroso al concepto medieval de la representación particularista y del mandato operativo, como las de los neo-proporcionalistas, que consideraban en cambio que la innovación proporcional no produce ningún efecto sobre la concepción de la representación política. Ambrosini afirmaba disentir de ambas posiciones, porque consideraba que la representación proporcional suponía «un sistema ecléctico que permit[ía] la aplicación simultánea, naturalmente sólo parcial, de principios y tendencias diversas" ${ }^{45}$.

La solución proporcionada por el constitucionalista de Favara era, por tanto, la de considerar a los partidos expresión de intereses generales y no particulares, de modo que la representación proporcional "[fuera] conciliable con el concepto llamado moderno de representación política» ${ }^{46}$. Pero Ambrosini refutaba la posibilidad, sostenida por parte de la doctrina (que no menciona de modo explícito), de conciliar la esencia de la concepción de la representación clásica con la de la nueva realidad institucional de la sociedad de masas. En efecto, evidenciaba con fuerza cómo en la relación representativa la entrada del partido, si no volvía a llevar al particularismo medieval, en todo caso introducía un tercer elemento.

En la conceptualización de Ambrosini se encuentran elementos que serán a continuación desarrollados por Gerhard Leibholz en la doc-

43 V. G. AMBrosinı, Partiti politici e gruppi parlamentari dopo la proporzionale, cit., pp. 5-6.

44 Ibídem, pp. 63 ss.

$45 \quad$ lbídem, p. 64.

$46 \quad$ lbídem, p. 65. 
trina alemana, con la afirmación de que el nuevo tipo de elección «confiere a los diputados la cualidad preeminente de representantes de los respectivos partidos, y los constriñe, aún sin llegar al mandato imperativo, a mantener con éstos vínculos estrechos de colaboración y de dependencia también después de las elecciones ${ }^{47}$. De modo más claro y utilizando un adverbio Ambrosini considera que «los diputados ya no pueden considerarse genéricamente como representantes de la voluntad de la nación, ni, menos aún, de su propia voluntad, sino que deben considerarse prevalentemente como representantes de la voluntad de los electores organizados en partidos" 48 .

En sustancia Ambrosini observaba y criticaba, pero a nivel teórico, en la obra de 1921 no quería y no podía formular una solución estable. Si es en efecto evidente la conciencia de la insuficiencia de la teoría clásica de la representación, es necesario observar que en 1921 las convulsiones del ordenamiento liberal-democrático no habían llegado a su cima de modo que las alternativas corporativas y de Consejos eran tomadas en consideración de manera integradora o para negarlas.

Por lo que se refiere a la institución monárquica, precisamente en 1922 sale el volumen de Emilio Crosa sobre La monarchia nel diritto pubblico italiano ${ }^{49}$ que puede ser considerada como la reconstrucción clásica de la posición de la Monarquía en el ámbito del ordenamiento constitucional italiano en la época de la crisis de participación liberaldemocrática y antes del fascismo. Emilio Crosa, discípulo también él como Ambrosini de Francesco Ruffini y de Gaetano Mosca, era autor considerado próximo a la Casa Real y había enseñado en la Escuela de aplicación de Turín. En el volumen rechazaba las interpretaciones de la doctrina alemana del principio monárquico y subrayaba con fuerza el cambio acaecido con la concesión del Estatuto en 1848. Esto -en su opinión- había reordenado de forma acabada la organización del Estado y, sobre la base de la falta de distinción entre leyes constitucionales y leyes comunes, había atribuido al Parlamento el "poder de legislar también respecto de las instituciones fundamentales del Estado, reduciendo por consiguiente el valor de la concesión al de mero expediente idóneo para instaurar el régimen constitucional de

$47 \quad$ lbidem, p. 68.

48 Ibídem, pp. 68-69.

49 V.E. Crosa, La monarchia nel diritto pubblico italiano, Torino, Bocca, 1922 obra que había sido precedida por La competenza regia nel diritto italiano, Torino, Bocca, 1916; sobre la obra de Crosa v., sintéticamente, la correspondiente voz en el Dizionario biografico degli italiani, dirigida por F. Lanchester. 
modo legal, determinando únicamente la continuidad histórica, que no jurídica, de la monarquia» ${ }^{50}$.

En un posterior ensayo sobre la concesión del Estatuto y la obra de Borrelli, que retoma ideas esbozadas precedentemente, el mismo Crosa evidenciará, con evidente nostalgia, la posición de la clase política saboyana que, fiel a la Corona y reconociendo las exigencias de los tiempos, aconseja al Soberano conceder el Estatuto y se retira a la sombra ${ }^{51}$. Pero en esta obra declara que "(era) necesario partir de la teoría jurídica del Estado moderno. Para ésta el Estado [era] persona jurídica, la soberanía [era] una cualidad jurídica del poder de imperio perteneciente al Estado" ${ }^{52}$, de modo que "el rey se presenta[ba] en nuestro derecho como órgano inmediato del Estado, órgano que por tanto recaba la propia existencia, como el ejercicio de las propias funciones de la misma constitución " ${ }^{53}$. En esta teorización del periodo terminal de la breve experiencia liberal-democrática el Rey no habría tenido, por tanto, prerrogativas, sino sólo competencias que lo limitaban jurídicamente. Se trata de una opinión coherente con las posiciones de la doctrina del periodo estatutario tardío, atenta a lo que será llamado el principio político, pero en este caso deliberadamente insensibilizada por la concepción de la personalidad estatal.

\section{LA TRANSICIÓN AL RÉGIMEN AUTORITARIO ENTRE VUELTA AL PASADO Y NUEVOS PARADIGMAS: LA CONTRIBUCIÓN DE TEODOSIO MARCHI}

El salto cualitativo en las reconstrucciones teóricas de la relación Monarca-Parlamento es, en cambio, identificable ya pocos meses después (enero 1923) en el discurso de apertura de Teodosio Marchi54 al 1922, p. 9 .

50 V. E. Crosa, La monarchia nel diritto pubblico italiano, Torino, Bocca,

51 E. CRosa, La concessione dello Statuto ed il ministro Borelli «redattore" dello Statuto (con lettere inedite di Carlo Alberto), Torino, 1936, y antes "Lo Statuto del 1848 e l'opera del ministro Borelli (con lettere inedite di Carlo Alberto)", en Nuova antologia, 16 de junio de 1915, pp. 533 ss.

52 Idem, pp. 12-13.

53 Ibídem, p. 16, v. también casi literalmente E. CROSA, voz "Monarchia", en Enciclopedia it. Treccani, vol. XXIII, pp. 616.

54 Teodosio Marchi, profesor y luego catedrático de Derecho constitucional en la Universidad de Urbino, enseñó en el Instituto universitario Cesare Alfieri de Florencia y en Macerata; acabó su carrera académica como catedrático de Derecho constitucional en la Universidad de Parma. 
Curso de Derecho constitucional en la Universidad de Macerata sobre /l Capo dello Stato nel governo parlamentare 55. Marchi, que en el pasado se habia ocupado significativamente por una parte de sanción y de procedimiento legislativo ${ }^{56}$ y por otro de lugartenencia ${ }^{57}$ y que abrazaba un punto de vista de tipo realista ${ }^{58}$ procedía a describir la crisis del ordenamiento como doble bloqueo de la función parlamentaria y de la gubernativa, con consecuencias sobre el mismo poder judicial. En tal situación "[pareció] - continuaba Marchi-que la misma Corona no conseguia sustraerse al fenómeno general, sufriendo a través de la responsabilidad de los ministros, los efectos de su titubeante acción, unas veces invadente otras tímida, sufriendo por tanto, dada la exquisita delicadeza de su posición, la disminución lenta gradual incesante de sus poderes» $\$$.

Después de la Marcha sobre Roma, Marchi intentaba justificar lo que había sucedido declarando que "[el] Jefe del Estado, el Soberano ocupa, en nuestro ordenamiento, el puesto central supremon 60 , por lo que en una monarquia parlamentaria el Jefe del Estado no debe representar «un cargo inerte, vano, destinado a ocupar un puesto que podría ocupar indistintamente otro".

La polémica jurídico política resulta evidente cuando Marchi justificaba a quien habia considerado "que [se podía] prescindir del consentimiento regio en algunos actos, que los Ministros del Rey [podían] anunciar al país una medida gravísima, nunca aplicada desde 1848, como era la proclamación del estado de sitio en todo el Reino, sin haberse cerciorado antes de la exacta intención del Jefe del Estado y sin tener su consentimiento" 61 .

Negándose a firmar el estado de sitio el Soberano habría - para Marchi- "destruido la leyenda, pesada en su ligereza como una pesa-

55 V. T. MARCHI, "ll Capo dello Stato nel governo parlamentare» en Rivista di diritto pubblico, 1923, 1, pp. 265 ss.

56 V. T. MARCHI, Sul concetto di legislazione formale, sanzione regia e rapporti fra Capo dello Stato e Camere, Milano, Società Editrice Libraria, 1911.

57 V. T. MARCH, Le luogotenenze generali (1848-1915) nel diritto costituzionale italiano, Roma, Athenaeum, 1918.

58 V. significativamente T. MARCH, "Lo Statuto albertino e il suo sviluppo storico", en Rivista di diritto pubblico, 1926, pp. 186 ss. (se trata del discurso de apertura de 1925 al Curso de Derecho constitucional en la Universidad de Parma). 266.

59 V. T. MARCHI, "ll Capo dello Stato nel governo parlamentare", cit, p.

$60 \quad$ Idem, p. 267.

61 lbidem, p. 269. 
dilla, según la cual el Rey debe limitarse a reinar, no puede y no debe gobernar" ${ }^{62}$.

Marchi expresaba, por tanto, de manera explícita el intento erróneo de una parte de la doctrina constitucionalista italiana de volver los acontecimientos ocurridos en octubre de 1922 a favor de un retorno a la monarquía constitucional, donde en el "gobierno democrático ... fuera garantizado un fuerte, vigoroso poder del Jefe del Estado ...»63. Se trata en parte de retomar la polémica contra el parlamentarismo, visto en su degeneración asamblearia, pero también contra el reforzamiento del Gobierno sobre la base de los decretos de 1876 y de 1900, con la petición - típica de las discusiones de los años noventa del siglo $\mathrm{XIX}$ - de la institución de un Consejo privado de la Corona sobre la base del modelo inglés ${ }^{64}$. La solución de Marchi era por tanto favorable a un Rey que reinase pero que no gobernase personalmente ${ }^{65}$, necesitando el Gobierno gozar de la doble confianza del Jefe del Estado y de las Cámaras.

En contraste con las posiciones de Ferracciù 66, que consideraba la transformación en sentido parlamentario fundada en la costumbre, Marchi consideraba que el Soberano, aun cuando irresponsable, debía ser responsable juridicamente ante el País y debía ser "verdaderamente órgano activo "67. Después de haber sostenido que la prerrogativa regia era la "máxima manifestación del poder político [y era] poder discrecional», solicitaba una reforma institucional que restituyera a las diversas instituciones "su justo valor" a fin de dar "a nuestra Constitución nueva juventud, al Principado nueva energía, a todos el sentido de una verdadera, de una sana, de una ordenada felicidad $"{ }^{68}$.

Me he detenido en la posición de Marchi porque es significativa de una parte de la doctrina liberal-conservadora, que por un cierto periodo se hizo la ilusión de poder gestionar los acontecimientos de 1922 del lado del poder del Jefe del Estado. En el discurso de apertura

62 Ibidem, p. 270.

63 Ibídem, p. 272.

64 V. C. Mozzarellu, "Un'autorità rebulosa come cirri». II dibattito di fine secolo sul Consiglio privato della Corona e le prerogative regien, en Cheiron, 1996, n. 25-26, pp. 41. Ss.

65 V. T. MARCHI, "ll Capo dello Stato ...", cit., p. 278.

66 V. A. Ferracciù, "A proposito di un libro recente su la riforma costituzionale", en Rivista di diritto pubblico, 1924, I, pp. 506 ss.

67 Idem, pp. 287-8.

68 Ibídem, p. 289. 
al curso universitario del mismo autor sobre Lo Statuto albertino ed il suo sviluppo storico, realizada en enero de $1925^{69}$ y fuertemente influida por la contemporánea obra de planteamiento liberal-nacional de Arrigo Solmi70, se percibe -en cambio- la conciencia de que el camino ya no es sólo el del sonniniano retorno al Estatuto, cuyos méritos radicaban en su forma escrita y en su flexibilidad. El problema que Marchi afronta es, por tanto, el de los límites a la modificabilidad del Estatuto, una carta en la que - afirma él- «hemos vivido, como pueblo libre e independiente ${ }^{71}$.

Más exactamente Marchi -anticipando un tema que Luigi Rossi propondrá en el curso del debate sobre las transformaciones institucionales de los años treinta72 - se planteaba el interrogante "sobre el punto hasta el cual nuestro poder legislativo ordinario puede avanzar en las mutaciones estatutarias; [la cuestión de] si siempre y en todo caso nuestro Parlamento puede constituir, él y sólo él, el órgano competente a esos efectos" ${ }^{73}$. Marchi negaba la omnipotencia parlamentaria sobre la base de la necesidad (Romano ${ }^{74}$ ) o de la costumbre (Ferraciù ${ }^{75}$ ) para identificar (citando a Dicey) cun conjunto de principios, de derechos, de instituciones fundamentales que constituye una barrera jurídica insuperable ${ }^{76}$. En este caso Marchi utilizaba una tesis, que había usado ya durante el trienio liberal-democrático post-bélico77, para sostener que «todos los ordenamientos constitucionales ... contienen una íntima fuerza de resistencia; representan un orden cerrado,

69 V. T. MARCHI, "Lo Statuto albertin oe il suo sviluppo storico", cit.

70 V. A. Solmi, La riforma costituzionale, Milano, Alpes, 1924. Arrigo Solmi, profesor de Historia del Derecho en las Universidades de Camerino y Cagliari, fue catedrático de la misma materia en Siena, Parma y Pavia. Terminó su carrera universitaria como catedrático de Ciencia política en la Facultad de Derecho de la Universidad de Milán.

71 V. T. MARCHI, "Lo Statuto albertino e il suo sviluppo storico", cit., p. 186.

72 V. L. RossI, "La elasticità dello Statuto", en Studi in onore di S. Romano, Padova, Cedam, 1940, pp. 25 ss. Luigi Rossi, catedrático de Derecho constitucional en la Universidad de Boloña, fue diputado y Ministro en el periodo liberal. Durante el fascismo fue el primer catedrático italiano de Derecho público comparado en la Facultad de Ciencias politicas de la Universidad de Roma.

73 V. T. MARCHI, "Lo Statuto albertino e il suo sviluppo storico", cit., p. 192.

74 Santi Romano, profesor de Derecho constitucional en la Universidad de Camerino, fue catedrático de dicha materia en Ródena, Pisa, Milán y Roma. Fue Presidente del Consejo de Estado.

75 Antonio Ferraciù fue catedráticco de Derecho constitucional en las Universidades de Siena y Palermo.

76 Idem, p. 193 ss.

77 V. T. MARCHI, La cosiddetta onnipotenza parlamentare, Cagliari, 1921. 
dentro del cual los órganos legislativos, extraordinarios y normales, pueden, jurídicamente, actuarn ${ }^{78}$.

Los límites a la revisión de la Ley fundamental de la Monarquía eran para Marchi la forma unitaria del Estado de tipo monárquico representativo, con una determinada monarquía, división de los poderes, sistema bicameral, responsabilidad ministerial, principio de libertad79. Desde esta específica perspectiva, Marchi invoca la vieja idea de un retorno a la "forma monárquica parlamentaria en su forma pura, no en las formas degenerativas que ésta ha venido asumiendo: forma monárquico parlamentaria [y] no simplemente constitucional' ${ }^{80}$. Se trataba de la recuperación de la idea del acuerdo entre Corona y Pueblo, de modo que el Gobierno debía moverse en la perspectiva dualista de mantener "la constante armonía" entre los mismos y cuando eso no fuera posible el Príncipe, supremo moderador de la vida del Estado" tenía a su disposición los instrumentos de la prerrogativa ${ }^{81 .}$

En este contexto Marchi, que representa en realidad el pasado, consideraba que las innovaciones institucionales podían concretarse en la reforma del Senado; en la eliminación de toda iniciativa parlamentaria en materia de gasto; en la garantía de independencia de la magistratura en el ejercicio de sus funciones, pero sobre todo que era necesario una vuelta a los principios que estaban en la base del Estatuto.

\section{SIN VUELTA ATRÁS: EL DESARROLLO DEL RÉGIMEN Y EL PAPEL DE LA MONARQUÍA}

La situación, sin embargo, había cambiado profundamente y pondrá en evidencia cómo las interpretaciones de tipo monárquicoconstitucional no tenían concreto fundamento en la nueva situación. Las discusiones en el seno de la llamada Comisión de los Soloni, y antes incluso en el seno del PNF (Partido Nacional-Fascista) evidenciaron - sin embargo- posiciones profundamente diferentes, indicadoras del debate unido al desarrollo del Estado que caracterizará todo el periodo fascista82. El dualismo monárquico-fascista presentaba, en

78 V. T. MARCHI, "Lo Statuto albertin oe il suo sviluppo storico", cit., p. 193.

79 Idem, p. 197.

$80 \quad$ lbidem, p. 199.

81 Ibidem, pp. 201-2.

82 V. Presidenza del Consiglio dei Ministri, Relacioni e proposte della nuova commissione presidenziale per lo studio delle informe costituzionali costitui- 
efecto, elementos de continuidad en la polemica antiparlamentocrática de la segunda mitad del ochocientos de tipo bonghiano y sonniniano, pero sobre todo la inserción de elementos nuevos (por ejemplo el Gran Consejo del Fascismo y el papel del Partido), que no podían ser encubiertos con el agotado recurso a hipótesis institucionales tradicionales.

De parte de la tradición se situaba sustancialmente la ponencia Sobre las relaciones entre poder ejecutivo y poder legislativo, redactada en el ámbito de la Comisión del dieciocho por Domenico Barone. En ésta se subrayaba, en primer lugar, que las asambleas políticas no debian participar en el poder ejecutivo «ni colaborando directamente con éste ni concurriendo en la elección de aquellos a los que se confía éste». Se proseguía sosteniendo que "[las] relaciones entre el Gobierno y las Cámaras deben estar presididas por el principio según el cual aquél es órgano de la Corona y no del Parlamento", de modo que «el Gobierno mismo se debe presumir legítimamente investido del poder mientras no sea privado de la confianza del Rey". En este sentido, "árbitro de la situación [debía ser] siempre y en todo caso la Corona, a la que [debía] corresponder en exclusiva la revocación y el nombramiento de los Ministros»83.

En realidad, como observó el constitucionalista Gaetano Mosca en el curso de la discusión parlamentaria sobre el proyecto de ley sobre las atribuciones del Jefe del Gobierno a fines de 1925, el silencio sobre la permanencia de la relación de confianza no instituía una forma de gobierno monárquico constitucional de tipo alemán, como demostraba la ponencia que acompañaba al mismo proyecto. En ésta se declaraba explícitamente que el Jefe del Estado mantendría en el cargo al Jefe del Gobierno mientras mantuviese el apoyo del conjunto de fuerzas económicas, políticas y morales que le habian llevado al Gobierno ${ }^{84}$.

Semejante ambigüedad estuvo en la base del intenso debate sobre la reforma del Estado, que ocupó —aunque de manera distintaa los actores politicamente relevantes y a la doctrina iuspublicística italiana durante los tres lustros sucesivos, acompañando a las reformas

ta con decreto presidenziale 31 gennaio 1925..., Roma, Provveditorato generale dello Stato, Librería, 1925 (v. también la edición realizada en Firenze, Le Monnier, 1932).

83 V. los comentarios del mismo Mussolini a la ponencia de referencia, en A. Aquarone, L'organizzazione dello Stato totalitario, Torino, Einaudi, 1965, pp. 5758; para el texto v. pp. 350 ss.

84 V. Senato del Regno, XXVII legislatura, I sessione 1924-26, Discussioni, vol. IV, pp. 4373 ss. 
que desarrollaron el régimen. La ley sobre el Jefe del Gobierno (Ley $\mathbf{n}$. $2263 / 1925$ ) y la relativa a su facultad del poder ejecutivo de emanar normas jurídicas (Ley n. 100 de 1926) constituyeron los dos primeros pilares constitucionales de la nueva entidad85. Seguirá -excluyendo del análisis el tema corporativo- la Ley sobre la ordenación y atribuciones del Gran Consejo del Fascismo (Ley n. 2693/1928), que certificará hasta qué punto la situación era completamente distinta de una vuelta a la monarquia constitucional pura.

La doctrina iuspublicista italiana participó en el debate, pero sólo una parte minoritaria se orientó, sin embargo, hacia la transformación radical de los paradigmas metodológicos y de las instituciones, mientras otros continuaron encubriendo las novedades sobre la base de la continuidad con el pasado ${ }^{86}$.

Los constitucionalistas jóvenes, que en los años treinta se plantearon el tema de la teoría de la Constitución sin abandonar el cauce de la escuela publicista nacional, observaron las transformaciones del ordenamiento italiano y su equilibrio inestable conscientes de que no se podía volver atrás en el tiempo. Desde este punto de vista es oportuno hacer referencia, en lo referente al tema objeto de esta ponencia, a la obra con que se estrena Costantino Mortati, redactada bajo la dirección de Luigi Rossi y de Sergio Panunzio, para señalar que en ésta estaba muy presente el tema metodológico realista de las zonas grises del Derecho constitucional, pero sobre todo que estaba muy claro que el esquema dualista introducido por el Estatuto había dejado "abierta la cuestión del poder supremo, esto es del órgano que debía tener la dirección política del Estado ....187.

En el volumen son traídas a colación tanto las tesis sobre el uso legítimo de la prerrogativa por parte del Soberano para lograr el acuerdo entre Rey y Pueblo (Moncalieri), como el paso al parlamentarismo como fruto de costumbres ${ }^{88}$ en contraposición a la teoría de Romano

85 V. sobre esto E. CROSA, "La dottrina della delegazione di potestà legislativa materiale nel diritto italiano, francese, germanico. Prolusione al corso presso I'Università di Pavia", en Rivista di diritto pubblico, 1927, (estr., Tivoli, Mantero, 1927) y las sucesivas observaciones (recuperables en los Studi Lambert) de C. Scнміт, "L'evoluzione recente del problema delle delegazioni legislative", en Quaderni costituzionali, 1986, n. 3, pp. 536-550.

86 V. sobre esto F. LANCHESTER, I giuspubblicisti tra storia e politica, Torino, Giappichelli, 1998.

87 V. C. MORTATI, L'ordinamento del Governo nel nuovo diritto pubblico italiano, cit (cit. En la reimpresión, Milano, Giuffrè, 2000), p. 62.

88 Idem, pp. 62 ss. 
(necesidad) y con la posición de Raneletti89 contraria a la posibilidad de la costumbre en el Derecho público. Sin embargo Mortati, influido por la doctrina alemana y en particular por la obra de Schmitt, señalaba lúcidamente que el equilibrio entre Rey y Parlamento "no puede ser sino el reflejo de una situación necesariamente transitoria: el equilibrio de los poderes elude, más que resuelve, el problema de cualquier forma de gobierno, es decir el problema del órgano supremo»"90. Tal tesis, en conexión con las expresadas por Carl Schmitt en el periodo, divergía sensiblemente del planteamiento más aséptico de Emilio Crosa91, condicionada -en cambio- sustancialmente por la obra de Robert Redslob sobre la forma de gobierno parlamentaria92 y por la literatura francesa del periodo (Mirkine-Getzévitch y Burdeau).

Mortati, por una parte, interpretaba de modo correcto la vicisitud constitucional de Italia sobre la base de la progresiva marginación del Soberano como lógica consecuencia del sistema parlamentario, y recordaba cómo en el periodo 1919-20, las reformas de la ley de elecciones generales de 1919 y del reglamento interno de la Cámara de agosto de 1920 habían proporcionado la certificación formal de esa realidad. Se daba, por otra parte, perfecta cuenta de los defectos de la forma de gobierno parlamentaria adoptada en Italia, a la que los decretos sobre la presidencia del Consejo de 1876 y de 1901 no podían aportar modificación incisiva; defectos que antes al contrario terminaban por acentuar, siendo fruto de éstos. "La efectiva fuerza del primer ministro -en un régimen parlamentario- puede surgir - sostenía élsólo sobre la base de una disciplina de partido rígida y de la homogeneidad política de los miembros del Gabinete [,] tales elementos ... desaparecieron con la ampliación de la clase política y con el fraccionamiento de los grupos" ${ }^{93}$. De aquí la convicción de que "si el mal funcionamiento de las instituciones constitucionales tenía su raíz en la falta de las condiciones y de los presupuestos de hecho necesarios al régimen parlamentario" ${ }^{94}$ se debía "actuar en profundidad renovando

89 Oreste Raneletti, profesor de Derecho administrativo en la Universidad de Camerino, fue catedrático de la misma materia en Macerata, Pavia y Milán.

$90 \quad$ Ibidem, p. 65.

91 V. E. CROSA, Lo Stato parlamentare in Inghilterra e in Germania, Milano, Treves, 1929.

92 V. R. REDSLOB, Die parlamentarische Regierung in ihrer wahren und in ihrer unechte Form, Tübingen, Mohr, 1918 (luego vuelta a publicar y ampliada en francés en 1924).

93 V. C. MoRTATI, L'ordinamento del Governo ..., cit., pp. 71-72.

94 Ibídem, p. 72. 
todo el sistema de las relaciones entre Ejecutivo y Legislativo, sin por lo demás intentar resucitar - lo que habría sido poco duradero- instituciones del pasado, y sobre todo sin romper los nexos orgánicos entre Estado y sociedad" 195 .

No era todavía completamente clara a Mortati la solución de la relación entre Corona y Fascismo, pero demostraba ser consciente de que -al comienzo de los años treinta- el ordenamiento constitucional italiano se insertaba en el ámbito de un régimen autoritario de masas, caracterizado por la soberanía del Estado- por la extensión de las competencias estatales y por el ordenamiento corporativo.

La eliminación de la "subordinación de la voluntad regia a la del Parlamento "había -en opinión de Mortati- restituido el nombramiento del Jefe del Gobierno «a la libre (si bien no arbitraria) determinación del Soberano" 96 . La distancia de las visiones de tipo constitucional sostenidas por Marchi radicaba sobre todo en la explicitación de dos vínculos establecidos a la elección del Jefe del Estado. Con el primero, de naturaleza específica prevista por la Ley sobre el Gran Consiglio, el Rey resultaba condicionado formalmente "por la obligación ... de tener en consideración la designación del Gran Consejo del fascismo" que para Mortati tenía «el mismo fin de la que, en régimen parlamentario, emanaba del Parlamento en caso de crisis»97. Con el segundo, en cambio, el Soberano estaba vinculado - como sustancialmente había recordado antes Mosca- por la unecesidad de concordancia ... con la concepción fundamental del Estado, que le imprime a éste su carácter distintivo, anima con un mismo espíritu todas las instituciones y que, con expresión comprensiva, puede llamarse Régimen" 198 .

Mortati hace por tanto referencia a un framework conceptual capaz de orientar la interpretación constitucional, en un sentido parecido a la investigación del mismo Crosa ${ }^{99}$, pero a diferencia de este último, que en aquellos años estaba profundizando el tema central de las

\footnotetext{
95 Ibidem.

$96 \quad$ Ibidem, p. 85.

$97 \quad$ Ibidem, p. 88.

98 Ibidem, p. 90.
}

99 V. E. CRosA, "Osservazioni sui principi generali come fonte del diritto pubblico", en Memorie dell'Istituto di esercitazioni nelle scienze giuridico-politiche dell'Università di Torino, Torino, Bocca, 1926 y «ll fattore politico e le costituzionin, en Studi di diritto pubblico in onore di O. Ranelleti, Padova, Cedam, 1931, pp. 149 ss. 
formas de Estado y de gobierno ${ }^{100}$ intentará fundar su propio enfoque no tanto en principios dogmáticos deducidos de la hipostatización de valores históricamente consolidados, cuanto de la fuerza o de las fuerzas políticamente relevantes en el interior del contexto histórico-social de referencia.

El volumen de Mortati, publicado en 1931 pero fruto de la tesis en Ciencias Políticas leída en 1929, evidencia de manera clara las novedades de la concepción del autor (que se consolidará en los años sucesivos hasta llegar a completarse con la formulación de la Constitución en sentido material) pero representa también la naturaleza in progress de una situación constitucional altamente inestable. En tal marco, en los años sucesivós, la aclaración del papel cada vez más incisivo del partido y la tendencia del régimen y de una parte de la doctrina a desarrollar los aspectos totalitarios del ordenamiento, hicieron que las funciones ejercidas por el Jefe del Estado fueran contempladas como un poder liminar, idóneo para salvar el conjunto del ordenamiento incluso a costa del mismo régimen.

\section{CONCLUSIONES}

El análisis de las posiciones asumidas por la doctrina durante el fascismo respecto del papel de la Corona y el estado del compromiso diárquico excede del objeto de esta ponencia. Como conclusión de estas notas, como jurista positivista debo observar que, en la dinámica institucional contemporánea existen algunas constantes que trascienden las mismas formas de Estado y los regímenes y que dan actualidad al debate seguido en estas páginas. En efecto, la doctrina iuspublicista de la segunda posguerra ha llevado a cabo reconstrucciones de la figura del Jefe del Estado en nuestro ordenamiento republicano precisamente sobre la plantilla de las posiciones señaladas en el periodo de la crisis de participación de los años veinte. El constitucionalista no puede, por tanto, prescindir de hacer referencia a ellas para ejercer una actividad interpretativa equilibrada relativa al Estado y a la dinámica de las relaciones entre los órganos constitucionales supremos.

Sobre la base de las opciones realizadas por el Constituyente con el orden del día Perassi, interpretaciones consolidadas incluso a nivel

100 V.E. CRosA, "Sulla teoria delle forme di Stato", en Rivista internazionale di filosofia del diritto, 1931, n. 1, pp. 18 ss y "Sulla classificazione delle forme di governo", en Scritti in onore di Santi Romano, cit., I, pp. 441 ss. 
internacional de la forma de gobierno parlamentaria de tipo monista o dualista se han superpuesto, de hecho, a la dinámica concreta de las relaciones políticas italianas del periodo republicano, volviendo a proponer argumentaciones orientadas a subrayar un mayor o menor relieve de la figura del Jefe del Estado.

En el primer lustro de los años cincuenta, algunas de las interpretaciones más autorizadas sobre el papel del Presidente de la República en nuestro ordenamiento fueron sostenidas por constitucionalistas como Emilio Crosa ${ }^{101}$ y por Teodosio Marchi ${ }^{102}$ en continuidad con una reflexión sobre los cometidos ejercidos por el mismo en continuidad con la experiencia estatutaria, mientras la inestabilidad de un sistema parlamentario caracterizado por formaciones antisistema alentó, además de interpretaciones monistas con prevalencia de la asamblea o del Gobierno ${ }^{103}$, un complejo de posiciones en las que se atribuía al Jefe del Estado la posibilidad de "transformarse, en caso de crisis del sistema parlamentario, de Jefe formal en jefe sustancial del Estado" 104 .

El poder de resolver las crisis no se basaba ya en la prerrogativa, sino en la función schmittiana de guardián de la Constitución. Durante los años cincuenta, tras la articulación casi británica de las relaciones institucionales entre los supremos órganos constitucionales del periodo degasperiano, la crisis de la mayoría centrista y las tensiones provocadas de la formulada apertura de la izquierda acabaron, por tanto, reforzando la exigencia de recuperar un árbitro o un garante del mismo ordenamiento político constitucional. Los acontecimientos sucesivos y la influencia de la experiencia francesa demostrarán la persistente actualidad teórica y pragmática de tal discusión, pero también su marcado carácter polémico.

101 V. E. CrosA, "Gli organi costituzionali e il Presidente della Repubblica», en Rivista trimestrale di diritto pubblico, 1951, pp. 91 ss.

102 T. MARCHI, "ll Capo dello Stato", en Comentario sistematico alla Costituzione italiana, dirigido por P. Calamandrei y A. Levi, Firenze, Barbera, 1959, II, pp. 105 ss.

103 Para un análisis de conjunto de las posiciones v. V. CRISAFULLI, "Aspetti problematici del sistema parlamentare in Italia", en Studi in onore di E. Crosa, Milano, Giiuffrè, 1960, I, pp. 621 ss.

104 V. C. Esposito, "ll Capo dello Stato parlamentare», en Studi in onore di E. Crosa, cit., I, p. 780. 